\title{
Determining important parameters related to cyanobacterial alkaloid toxin exposure
}

\author{
A. H. Love
}

September 19, 2005

International Symposium on Cyanobacterial Harmful Algal Blooms (ISOC-HAB)

Durham, NC, United States

September 6, 2005 through September 10, 2005 
This document was prepared as an account of work sponsored by an agency of the United States Government. Neither the United States Government nor the University of California nor any of their employees, makes any warranty, express or implied, or assumes any legal liability or responsibility for the accuracy, completeness, or usefulness of any information, apparatus, product, or process disclosed, or represents that its use would not infringe privately owned rights. Reference herein to any specific commercial product, process, or service by trade name, trademark, manufacturer, or otherwise, does not necessarily constitute or imply its endorsement, recommendation, or favoring by the United States Government or the University of California. The views and opinions of authors expressed herein do not necessarily state or reflect those of the United States Government or the University of California, and shall not be used for advertising or product endorsement purposes. 


\title{
Determining important parameters related to cyanobacterial alkaloid toxin exposure
}

\author{
Love, A.H. ${ }^{1}$ \\ ${ }^{1}$ Forensic Science Center, Lawrence Livermore National Laboratory, P.O. Box \\ 808, L-178, Livermore, CA 94550
}

\section{Introduction}

The United States is faced with having to address critical issues that have not been addressed in the past, as recent security interests have placed new and more pressing demands on the assessment of risk from exposure to potential threats resulting from deliberate contamination. Such assessments are the basis for decisions about future research priorities, actions taken to prevent intentional contaminant releases, and developing detailed plans for response if such an event was to occur. High fidelity assessments are based on robust knowledge of the numerous parameters that can be used to predict the fate, transport, persistence, and toxicity for contaminants under numerous circumstances. Therefore, identification and determination of these parameters are the primary steps for evaluation of potential threats.

Numerous toxic substances exist, but very few have the potential to cause mass casualties from widespread contamination. Conventional chemical and biological weapons have strict laws that control their possession, transport, and use. Military studies of conventional agents have identified and determined many of the parameters necessary for accurate threat assessments. Spanning the threat space between chemical and biological weapons are biotoxins. Biotoxins are chemical toxins that are of natural biological origin. These compounds also have unique physio-chemical properties compared to other chemical and biological agents. Most natural toxins have few controls over possession, transport, and use; thus, these biotoxins have greater potential availability than conventional threat agents. They can range from large proteins (10s to $100 \mathrm{~s} \mathrm{kDa}$ ) to small molecular weight molecules ( 100- 500 MW). Large biotoxins must be extracted from biological material, but the small molecular weight toxins can be obtained either through biological extraction (i.e. Barros et al., 2004) or through methods for direct chemical synthesis published in scientific journals (i.e. Kishi et al., 1977); Jacobi et al., 1984; Mansell, 1996).

Cyanobacteria produce some of the most potent biotoxins known. Many of these cyanobacterial toxins (cyanotoxins) have low-level acute toxicity that are comparable to the toxicity levels from conventional chemical weapons and therefore warrant consideration as a potential threat (Burrows and Renner, 1999). The alkaloid class of cyanotoxins contains three types of the toxins considered here; saxitoxins (STX), anatoxins (ATX), and anatoxin-a(S). As alkaloids, each of these compounds are small molecular weight organic 
compounds with basic functional groups and a heterocyclic ring containing at least one nitrogen atom (Figure 1 ). These toxins are rapidly acute neutrotoxins, although the toxicological mode of action is different for each type. STX is a tightly regulated biotoxin because of its listing on the Chemical Weapons Convention Schedule 1 list. Possession, transport, and use of ATX and anatoxin$\mathrm{a}(\mathrm{S})$ are unregulated. The increasing occurrence of cyanotoxins in freshwater drinking supplies, as a result of increasing algal bloom containing cyanobacteria, have resulted in "Cyanobacteria and its toxins" listed on the US EPA Drinking Water Candidate Contaminate List 2 in February 2005. Other than acute toxicity levels, our current understanding of alkaloid cyanotoxins is limited and prevents an accurate assessment of whether these toxins pose a legitimate threat.

\section{Model and Parameters for Assessment of Potential Threats}

Since cyanobacteria naturally release alkaloid toxins into freshwater sources, numerous pathways of exposure are being considered in order to assess the information requirements for potential water quality regulations. A schematic model for any general assessment and the links between different components are shown in Figure 2. Most of the parameters needed for an assessment of a natural cyanotoxin release are also applicable to an intentional cyanotoxin release. A greater number of these model parameters have been determined for microcystin, a cyclic polypeptide liver cyanotoxin, since it is easier to measure quantitatively due to an aromatic ring in its structure that permits UV detection. Recognizing the need for greater protection from these naturally produced toxins, the $\mathrm{WHO}$ and several countries have established water quality regulations for microcystin based on conservative assumptions using this greater but still limited scientific understanding. Although assessment of the potential risk from intentional alkaloid cyanotoxin contamination has considerable overlap with natural releases, below is a discussion of some additional pieces of information that would be useful to know in order to address issues specific to intention releases.

\section{Source}

The starting point for any contaminant model of exposure is characterizing the magnitude and duration of the source of contamination. The information about the source affects all other parts of the exposure model. For natural releases, environmental factors and the biological community that result in cyanotoxin production limit the source characteristics, but intentional releases do not have the same source constraints. Thus, the potential is greater for the source of intentional contamination to reach acute concentrations than under natural conditions. For intentional contamination there is unique information that would be useful to be able to characterize about the source, such as the method of cyanotoxin acquisition and any attribution signatures. Identification of other chemicals associated with the intentional release that indicate if the toxins were synthesized directly or extracted from algal blooms provides important information about the technical abilities of the party involved with an intentional 
release. Other attribution signatures that can result from related temporal, spatial, physical, chemical, biological, or environmental information which can be used to narrow the identification of the responsible party is also a desirable capability for characterizing the cyanotoxin source.

\section{Fate and Persistence Parameters}

Once released, a mechanistic understanding of how cyanotoxins interact with their environment permits some prediction of how long these toxins persist in their environment and in what matrix the toxins are expected to reside. The primary parameters that can be used construct a mechanistic understanding are 1) flow conditions, 2) conditions and rates for degradation, 3) adsorption/complexation reactions and the affects of such associations on degradation and transport, and 4) enhanced degradation mechanism, such as biodegradation and catalysis. Little quantitative research has been performed to determine these parameters, although there have been some previous studies that indicate some general properties of some of these alkaloid cyanotoxins:

- Both STX and ATX are stable under acidic conditions, labile under alkaline conditions, and resistant to oxidation by $\mathrm{HOCl}$ (Burrows and Renner, 1999).

- STX is thermally labile at normal temperatures (Burrows and Renner, 1999; Indrasena and Gill, 2000);

- ATX is photolabile on the order of hours to weeks (Stevens and Krieger, 1991).

- Little research on fate and persistence has been performed for anatoxin$\mathrm{a}(\mathrm{S})$, but it has structurally similarities to existing organophosphorous pesticides, such as glyphosate, where most of the fate and persistence parameters are well-established (Rueppel et al., 1977).

Thus, models for the assessment of these cyanotoxins require a quantitative and mechanistic understanding of environmental interaction for a quantitative assessment and prediction of the potential threat for conditions not specifically tested in the laboratory.

\section{Exposure Matrices}

The exposure matrices for intentionally released cyanotoxins overlap with the matrices currently being considered for natural cyantoxin occurrences. Surface water bodies that are used either for recreation or for drinking water sources are the primary exposure matrix receiving consideration for natural releases and can represent either an ingestion or dermal exposure (Burrows and Renner, 1999). Other natural exposure matrices receiving consideration are 1) aerosols generated during irrigation or showering and 2) contaminated crops, either within plant cells or as surface contaminants, on agricultural products irrigated with contaminated water. Aerosols represent an inhalation exposure, whereas contaminated crops represent an ingestion hazard. Different exposure pathways may have significantly different levels of toxicity and exposure matrices may affect the bioavailability of the toxins. Our current knowledge of important exposure matrices and the difference between different exposure pathways is 
limited, but is an important component for development an overall assessment of the potential threat of these toxins.

\section{Uptake Parameters}

Very little is known about how exposure pathways and uptake dynamics affect the overall toxicity of alkaloid cyanotoxins. This is where the greatest difference exists between natural and intentional cyanotoxin contamination. Natural releases are more likely to pose a long-term exposure/uptake, whereas intentional releases are more likely to pose a short-term exposure/uptake. These fundamental differences are what result in the emphasis of acute toxins over chronic toxins for intentional contamination assessment consideration.

\section{Dose Characterization and Toxicity Parameters}

The acute toxicity levels of these alkaloid toxins are well-established for mice and there is information about human toxicity resulting from STX exposure in shellfish. As stated above, the effective dose that results from bioavailability modification of the exposure matrix may have a significant affect of the actual toxicity. Presently, the toxicokinetic and toxicodynamics information is limited and does not allow for a mechanistic understanding of human toxicity. Although no long-term adverse affects have been observed from sub-lethal exposure, there has not been any determination if there is any biological response that may result in the detection of a biomarker or interference with sensitive cellular function, such as prenatal development.

\section{Health Effects}

The ultimate endpoint for any threat assessment from an intentional contamination is the human health impact. Such an assessment may be significantly different for natural vs. intentional exposure since magnitude and duration of the different sources may have important differences. Because dose and toxicity information is limited, estimates of deaths, hospitalizations, and any long-term health effects have large uncertainties. A better understanding of dose and toxicity mechanisms would permit better estimates of human health impacts and may allow for the identification of improved methods for diagnosis and treatment. Implementation of the complete assessment model should assist in determining if a set of conditions exist where these toxins pose a legitimate public health threat, and if so, understanding the spatial distribution and total number of health impacts for both natural and intentional contamination scenarios.

\section{Model Impacts for Homeland Security Management}

Accurate modeling predictions are needed for a high-fidelity threat assessment, but also the model information is needed as a tool for management of the potential risk. An understanding of the issues related to an intentional release of these toxins is a prerequisite for proper preparation and an appropriate response to such an event. A typical diagram for the management of Homeland Security 
threats is shown in Figure 3. Prior to an incident of national security there are "Prevention \& Preparation" steps to mitigate the impact of intentional contamination events. During and after a contamination event there is an "Assessment \& Response" Phase followed by a "Decontamination and Recovery" Phase. Insight gained from a thorough assessment will result in improved consequence management to such an event.

\section{Prepare \& Prevent}

Source material management and monitoring are the primary means of mitigating the impact from an intentional biocontamination event. For cyanotoxins that pose a legitimate threat, appropriate quantities necessary for legitimate research purposes (i.e. understanding natural cyanotoxins occurrence and affects or pharmaceutical applications) need to be established without hindering the advancement of science. As with other highly toxic materials, possession of quantities of alkaloid cyanotoxins that the assessment model implicates as a legitimate threat can be tracked and inventoried. Currently methods for detection and quantification of these toxins require highly trained scientists, and sensors for these toxins do not exist. Monitoring using periodic sampling is likely to be more effective for natural cyanotoxin releases, where exposure tend to be chronic. For acute exposure from intentional releases, continuous monitoring is the only way to provide any degree of health protection for short duration exposures. These criteria are part of numerous existing efforts to understand how to best implement Early Warning Systems to the numerous potential threats of intentional contamination. Fate and persistence information on these cyanotoxins is needed for establishing criteria for Early Warning Systems.

\section{Assessment \& Response}

After an event where alkaloid cyanotoxins were intentionally released, the recognition and identification could result from either monitoring alerts or significant changes in public health status. In either case, identification of these toxins as the contaminate source is critical to effect triage and prevent additional exposures soon after the initial recognition. Clinical toxin identification procedures for legitimate cyanotoxin threats, established through the toxicological parameters of the threat assessment model, should be established for early diagnosis and analytical confirmation methods for important exposure matrices are additionally needed. Once the toxin is identified, a number of responses are necessary to minimize any additional exposure, including identification and elimination of the contaminant source, infrastructure modification to prevent further exposure, forensic sampling and analyses, and public notification. In some cases, the source may not be readily known and using the information collected an event reconstruction can be performed to identify the location and characteristics of the contaminate source. Any event reconstruction will be based on threat assessment model parameters that are then utilized to execute the model backwards (Figure 1) so exposure and health effects information can be used to determine source characteristics. For such efforts to be successful, high-fidelity data and models are required. 
Decontamination \& Recovery

Safe reoccupation/utilization is the goal after a biocontamination event. Efforts for recovery of critical infrastructure affected by the intentional contamination may require active decontamination if the agent doesn't naturally degrade over time. Methods for decontamination can utilize information about the degradation, biodegradation, and catalysis of alkaloid cyanotoxins determined for the threat assessment. The toxicological information from the threat assessment could be valuable in establishing public health standards for decontamination also. A basic tenet of Recovery is risk communication which includes educating the stakeholders of the risks associated with the incident, decontamination efforts, and safe re-occupation of decontaminated sites.

\section{Conclusions}

Science-based decision making required robust and high-fidelity mechanistic data about the system dynamics and impacts of system changes. Alkaloid cyanotoxins have the characteristics to warrant consideration for their potential threat. Since insufficent information is available to construct a systems model for the alkaloid cyanotoxins, STX, ATX, and anatoxin-a(S), an accurate assessments of these toxins as a potential threat for use for intentional contamination is not possible. Alkaloid cyanotoxin research that contributed to such a model has numerous areas of overlap for natural and intentional health effects issues that generates dual improvements to the state of the science. The use of sensitivity analyses of systems models can identify parameters that, when determined, result in the greatest impact to the overall system and may help to direct the most efficient use of research funding. This type of modeling-assisted experimentation may allow rapid progress for overall system understanding compared to observational or disciplinary research agendas. Assessment and management of risk from intentional contamination can be performed with greater confidence when mechanisms are known and the relationships between different components are validated. This level of understanding allows highfidelity assessments that do not hamper legitimate possession of these toxins for research purposes, while preventing intentional contamination that would affect public health. It also allows for appropriate response to an intentional contamination event, even if the specific contamination had not been previous considered. Development of science-based decision making tools will only improve our ability to address the new requirements addressing potential threats to our nation.

This work was performed under the auspices of the U.S. Department of Energy by University of California, Lawrence Livermore National Laboratory under contract W-7405-Eng-48. 


\section{References}

Barros, LPC; Monserrat, JM; Yunes, JS. (2004) Determination Of Optimized Protocols For The Extraction Of Anticholinesterasic Compounds In Environmental Samples Containing Cyanobacteria Species. Environmental Toxicology And Chemistry 23 (4): 883-889.

Burrows, WD; Renner, SE. 1999. Biological Warfare Agents As Threats To Potable Water. Environmental Health Perspectives 107 (12): 975-984.

Indrasena, WM; Gill, TA. 2000. Storage Stability Of Paralytic Shellfish Poisoning Toxins. FOOD CHEMISTRY 71 (1): 71-77.

Jacobi, PA; Martinelli, MJ; Polanc, S. (1984) Total Synthesis Of (+/-)-Saxitoxin. Journal Of The American Chemical Society 106 (19): 5594-5598.

Mansell, HL. 1996. Synthetic Approaches To Anatoxin-a. TETRAHEDRON 52 (17): 6025-6061.

Rueppel, Ml; Brightwell, Bb; Schaefer, J; Marvel, Jt. 1977. Metabolism And Degradation Of Glyphosate In Soil And Water. Journal Of Agricultural And Food Chemistry 25 (3): 517-528.

Stevens, Dk; Krieger, Ri. 1991. Stability Studies On The Cyanobacterial Nicotinic Alkaloid Anatoxin-A. Toxicon 29 (2): 167-179.

Tanino, H; Nakata, T; Kaneko, T; Kishi, Y. 1977. Stereospecific Total Synthesis Of D,L-Saxitoxin. Journal Of The American Chemical Society 99 (8): 28182819 . 
Figure 1 - Structure of alkaloid cyanotoxins; A) saxitoxins B) anatoxins, C) anatoxin-a(S)

A

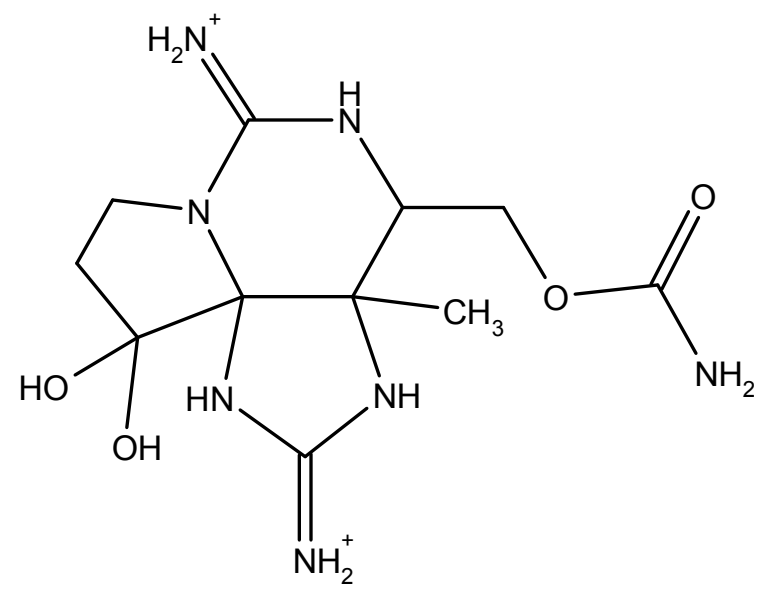

B

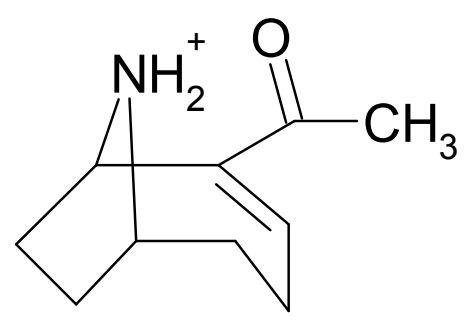

C<smiles>COP(=O)([O-])ON1C(=[NH2+])NCC1CN(C)C</smiles> 
Figure 2 - Schematic Model of Information Needed for Threat Assessment

\section{Source}

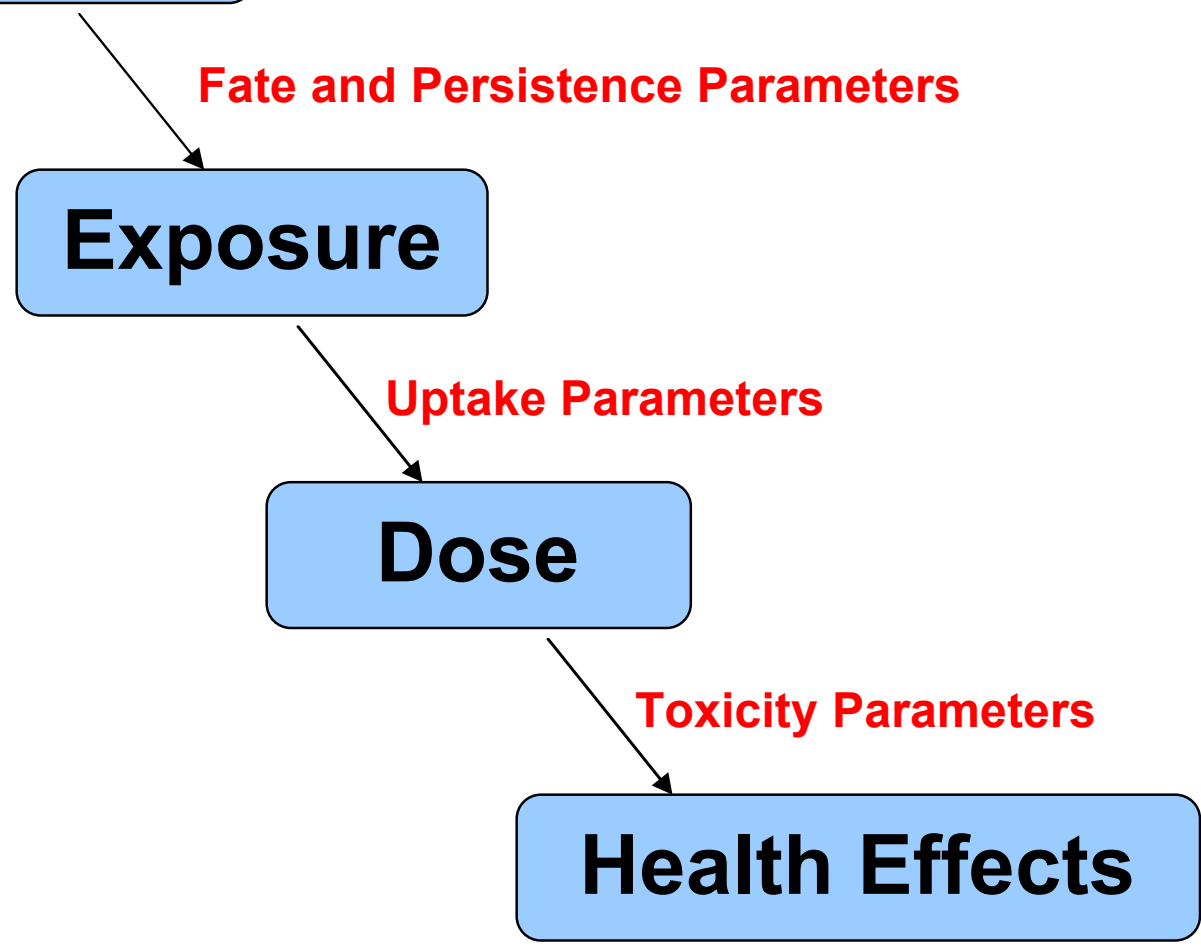


Figure 3 - Simplified Management Perspective of Homeland Security Threats

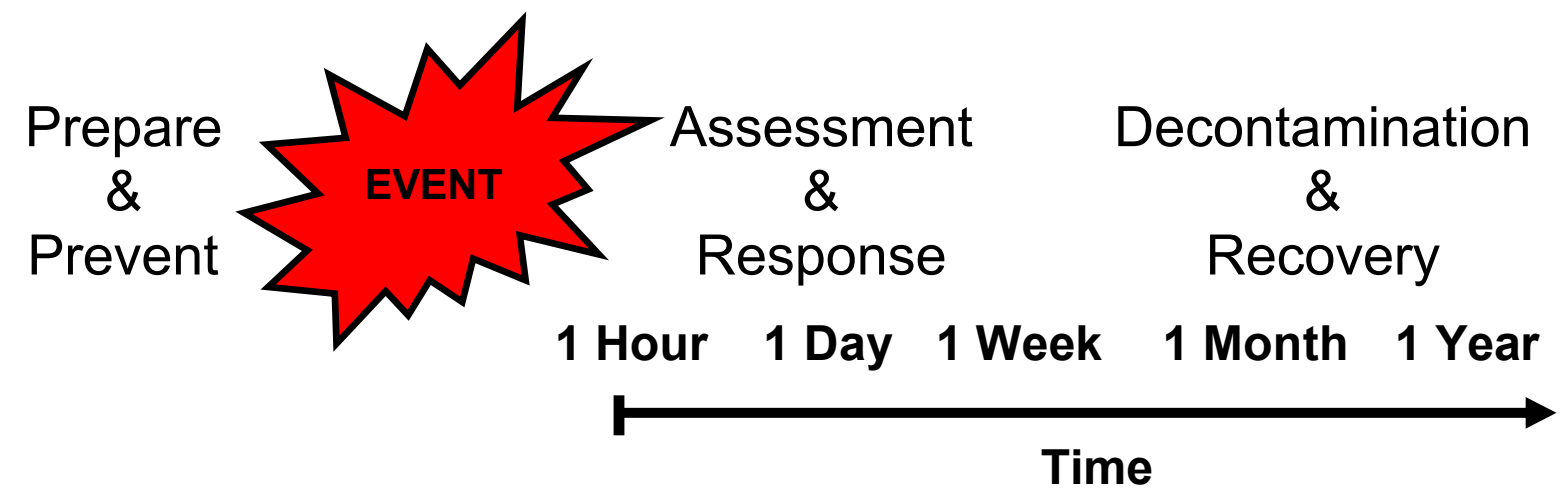

